\title{
Metabolism-Schrodinger the Sixth Physical Field
}

\section{Bortz WM*}

Stanford University, School of Medicine, Portola Valley, CA 94028, USA

\section{Introduction}

Metabolism represents the nexus of fundamental physical forces, which while present in all structure and function require new explanatory emergent principles, which, so far, cannot be predicted or derived solely from description of chemistry and physics. Metabolism is essentially concerned with the transduction of energy flows with respect to time, space, and matter. Language models and metaphors contribute to construction of scientific explanation within biology. The concept of a metabolic field yields a deeper, broader, more quantitative integrated theoretical framework leading to novel predictive models.

Since the dawn of civilization language has been the concourse for mankind. It is humanity's basic tool. Conflict, resolution, communication, politics, trade, entertainment, economics, and certainly science, all human cognitive domains require language. Language represents the very core and essence of science. Austrian philosopher Ludwig Wittgenstein observed "the limit of my language means the limits of my world" [1].

Language is the vehicle for the creation and transmission of ideas. Grammar defines thought. Thought defines grammar. Freeman Dyson observed that scientific revolutions are driven by new ideas and by new tools [2]. The first sentence in Lewontin's book, The Triple Helix, asserts that it is impossible to do the work of science without the use of metaphors [3]. "Virtually the entire body of modern science is an attempt to explain in words phenomena that cannot be experienced directly." The metaphor helps to make complex phenomena comprehensible. The most far reaching of these was Descartes's conception of the world as a machine in 1630 . The image of a clockwork universe is an immediate derivative. This format gave birth to the mechanical view of the world and its contents, powerfully described in Newton's Principia, a metaphorical framework which extends until today [4].

Since prehistory mankind has strived to describe the elements of human existence in verbal terms. Greek scholars deserve much credit for removing metaphysics as an agency on which to base further understanding. Aristotle chided Homer for employing supernatural agencies as a heuristic in his classic tales. He suggested a knowledge domain based on experiment and empiricism. Mathematics and astronomy were products of early imagination and reasoning. The empiricism inherent in The Enlightenment led to chemistry and physics, the sciences required to propose an explanatory platform for the nature of life.

Their reductionistic techniques were successful in generating corollary insights, codified as the Field theories, which underlie most of physical science. Gravity, electromagnetism, the strong and weak nuclear forces, and more recently quantum fields are acknowledged global constructs.

The concept of a field seeks to place fundamental elements of matter, energy, space, and time into a coherent relationship with one another.

Historically, the use of the term "field" to explain a physical phenomenon is credited to Faraday, who in 1824 defined electromagnetism as a force with both time and space dimensions a field [5]. It implied an action, acting at a distance through an intervening medium, a field, a metaphorical representation of lines of force. Field theories use mathematical quantities such as numbers or vectors to describe how the conditions of any point in space will affect matter or another field.

Einstein's concept of fields differs in specifics, but the semantic differences do not obscure the basic metaphoric value of the term field, particularly as it accumulates data points derived from new knowledge of the biochemistry and biophysics of intermediary metabolism. The distinction between matter and energy is blurred. In 1938 Einstein wrote "the concept of the field is the most important invention since Newton. It needed great scientific imagination to realize that it is not the charges or the particles but the field between the charges and the particles that is essential for the description of physical phenomena" [6]. "The concept of the field is the greatest contribution to the scientific spirit" [7]. Isaacson wrote of Einstein's "Lifelong devotion to field theories as a way to describe nature" [8].

The success of the reductionist method which led to the Field Theories gave rise to the hope that a grand unified Theory of Everything would derive and allow penetrance into the nature of uni- and multicellular life by generating a set of equations capable of describing all phenomena ever observed and ever to be observed.

However, Laughlin and Pines assert that the proposed Theory of Everything is "irrelevant to important things in nature [9]. We have succeeded in reducing all of ordinary physical behavior to a simple, correct Theory of Everything, only to discover that it has revealed exactly nothing about many things of great importance. Reductionism has reached its limits". That which emerges is the need for a new way of seeing and/or thinking.

The recent rapid progress in the reach for a fundamental understanding of the physical laws for life derives directly from Schrodinger's three lectures at Trinity College in 1944 [10]. These presentations represent a breakthrough moment in the effort to understand the organic basis of life. Schrodinger, a quantum physicist, based his arguments on the Second Law of Thermodynamics, proposing that all life is an inevitable by-product of energy flow on matter, over time. Similar to Kant's dualism of "moving power" and "formative power" [11] Schrodinger famously divided his discussion into two parts, replication and metabolism. In his derivative book Origins of life Dyson faults Schrodinger for his granting replication the higher priority in the basic processes [12]. Dyson postulates that

*Corresponding author: Bortz WM, Clinical Professor of Medicine, Stanford University, School of Medicine,167 Bolivar Lane, Portola Valley, CA 94028, United States, Tel: 650-854-2273; E-mail: drwbortz@gmail.com

Received February 04, 2016; Accepted February 18, 2016; Published February 20, 2016

Citation: Bortz WM (2016) Metabolism-Schrodinger the Sixth Physical Field. Metabolomics 6: 169. doi:10.4172/2153-0769.1000169

Copyright: ( 2016 Bortz WM. This is an open-access article distributed under the terms of the Creative Commons Attribution License, which permits unrestricted use, distribution, and reproduction in any medium, provided the original author and source are credited. 
the reproduction/replication aspect of life's origin is satisfactorily understood in classical biochemical terms by the precise matching of complementary strands of nucleic acids.

Importantly the five classic fields of the scientific enterprise in Schrodinger's era gravity, magnetism, the strong and weak molecular forces, and quantum excluded Time from their domains. All were theoretically reversible and as such were not consistent with any comprehensive detailing of "What is Life?"

The vacuous term Negentropy was his feeble attempt at competence. Pauling credits Schrodinger's insight with the birthing of molecular biology, the advent of which has served to integrate biologic science with the details inherent in the rapidly maturing domain of physical law [13]. Recent decades have witnessed an avalanche of central new biologic knowledge; a Rosetta Stone of metabolism has been elaborated.

Dyson points out that scientific progress are generated by tools and by ideas [2]. The two tools that have fed the explosive increase in current knowledge are the computer and genetics. The computer has provided the hardware for the quantitative probing of the heart of matter. Genetics has provided the systematics of reproduction. The computer has provided a brilliant measuring instrument.

The adoption of genetics and the computer as the basic strategic tools for comprehensive understanding has in my view created a detour in our process of providing a new inclusive paradigm for biology. It has created a huge library of data concerning gene structure and function, dominated by generation of GWAS phenomenology [14]. This detour promised much but delivered little. Lewontin and many others comment on the insufficiency of the gene fixation [15]. The gene is not enough. It is an essential part, but insufficient in and of itself.

The dominance of GWAS fixation is now largely relieved by the simple description of its replacement EWAS, environment wide association study [16]. This inclusion of environment at the nature/ nurture interface appropriately acknowledges the imperative interaction. The new model of EWAS is much more complex and interconnected, but so is life. EWAS is the appropriate replacement for GWAS.

In our pursuit of deep knowledge the Schrödinger proposition creates a descriptive challenge as the explosive collection of relevant data points exceeds our grasp, the resultant ideas lag. Molecular biology overloads its descriptive capacity. Science requires adequate framing terminology. Language must serve technology.

An even more formidable issue is that which was explored by Richard Strohman in his Nature Biotechnology 2003 paper "Thermodynamics -old Laws in Medicine and Complex Disease" [17]. In seeking the new rules for life he observed "that the laws of thermodynamics are intimately linked to the phenotype of the organism through the agency of dynamical systems. Sadly this essential point has been all but ignored in the rush to find agent-based genomic-proteomic explanations. Looking back that substitution of agents for agency must be recognized as an epistemologic error of great moment." Switch from a dynamic approach to a static one represents a substitution of matter for process. Dynamics are excluded. Other implications that derive from this dynamic interpretation include the substitution of the term homeodynamics as an explanatory term for homeostasis [18], which upon any reflection is alien to life.

Such a cognitive excursion fails to recognize the central role played by time as a core element of biology. Life is of itself differentiated from the inorganic world that has little relation with time, but biology is defined by its historical context. It is in effect central and defining what life is.

A recent series of papers in the Lancet addressed the need for an interdisciplinary new biology. Among them Geoffrey West advocated for a "quantitative, predictive, multilevel, theoretical framework that both complements the present approaches and stimulates a more integrated research agenda that will lead to novel questions and experimental programmes" [19].

It is therefore with this sense of current inadequacy that I propose the terminology of a Metabolic Field-Schrodinger [20]. This provides a framework that integrates the classic physical laws within the vast data derived from contemporary molecular biology. The term provides a blueprint for the establishment of a new model for life. Provision of the term Metabolic Field -Schrodinger provides a tool to peer into the nature of complex adaptive matter, the biologic phenomenon intimately involving the laws of physics and beyond. Anderson's central paper, More is Different [21] simply recognizes that the basic tenets of inorganic matter and the derivative five field theories are time insensitive, and thus are not relevant to the openness and irreversibility of biologic process. The Metabolic Field-Schrodinger is differentiated from the prior classic physical laws by 1) being open, 2) acknowledging the free interplay of energy and matter 3) is historical, employing a time dimension 4) is cyclic 5) is dynamic, and stable until dissipating This is the platform that is exhibited by the Metabolic Field-Schrodinger, and hopefully provides a conceptual foundational framework for the inclusion of the emerging science domains. We need a new epistemological capacity which deals with the domain of the as yet undiscovered organizing principles and function of living organisms which grow out of the equations of microscopic rules but are independent of them.

The structure and function of biology find more complete description when considered as a part of the proposed rubric of the Metabolic Field-Schrodinger. Prior conceptualization has proven to be inadequate. No longer is consideration of agents deemed to be sufficient to describe life. Active agency replaces the notion of components. Life is a verb rather than a noun. The living system, not its parts, is the correct hierarchical level of inquiry and description. "More" is vastly wonderfully different! A Field.

\section{Conclusion}

I propose the introduction of a new epistemological term "Metabolic Field (Schrodinger)." Such language embraces the totality of the explosive increase in biologic science that has become so vast and complex that the phrase "catastrophe of complexity" has been applied to the domain. This new term integrates the classic physical fields with that of molecular biology seeking to provide a conceptual framework for biologic science.

\section{References}

1. http://www.brainyquote.com/quotes/authors///ludwig_wittgenstein.html.

2. Dyson F (2013) Is Science Driven by Ideas or by Tools? Science 339: 14261428.

3. Lewontin R (2002) The Triple Helix, Harvard Press, Cambridge, England.

4. Newton I (1687) Mathematical Principles of Natural Philosophy S. Pepys London.

5. Faraday M (1860) Course of Lectures on the Various Forces of Matter and their Relationships to each other.

6. Einstein A, Infeld H (1938) The Evolution of Physics: the Growth in Ideas from early Concepts to Relativity and Quanta. Cambridge University Press, New York, USA. 
7. Holton G (1973) Thematic Origins of Scientific Thought: Kepler to Einstein Harvard University Press, Cambridge, England.

8. Isaacson W (2008) Einstein: His Life and Universe. Simon and Schuster, New York, USA.

9. Laughlin W, Pines D (2000) Proc Natl Acad Science 97: 28-32.

10. Schrodinger E (1967) What is Life? The Physical Aspects of Living Cells. Cambridge University Press New York, USA.

11. Kant I (2004) Metaphysical Foundations of Natural Science Cambridge University Press, New York, USA.

12. Dyson F (2001) Origins of Life. Cambridge University Press New York, USA.

13. Pauling L (1981) Schrodinger Contributions to Chemistry and Biology in Schrodinger: Centenarian Celebration presentation Imperial College.

14. NIH Catalog of Published GWAS Studies 2015 Bethesda.
15. Lewontin R (2011) Even Less in our Genes New York Review Books May 20.

16. Patel CJ, Rehkopf D, Leppert J, Bortz W, Cullen M et al. (2013) Systematic evaluation of environmental and behavioural factors associated with all-cause mortality in the United States national health and nutrition examination survey. Int J Epidemiology 42: 1775-1810.

17. Strohman R (1993) Ancient Genomes, Wise Bodies, Unhealthy People; Limits of a Genetic. Paradigm Perspec Biol Med 37: 112-145.

18. Bortz WM (2015) Updating Homeostasis. Biol syst Open Access 4:138.

19. West GB (2012) The Importance of Quantitative-System Thinking in Medicine. The Lancet 379: 1551-1559.

20. Bortz W (2012) Metabolic Field -Schrodinger An Explanatory Platform for Biology Based on Lecture at Trinity College, Dublin, Ireland, July 18, 2012. Med Hypotheses 85: 894-897.

21. Anderson P (1972) More is Different. Science177: 393-396. 